\title{
Studies and realization of an experimental set-up for micro Airy beams generation
}

\author{
A. Mathis, M. Jacquot (*), F. Courvoisier, L.Froehly and J.M. Dudley \\ FEMTO-ST Institute, Department of Optics P.M. Duffieux, UMR CNRS 6174, Université de Franche-Comté, \\ 25030 Besançon, France \\ (*)maxime.jacquot@univ-fcomte.fr
}

Airy beams are a solution to the paraxial wave equation with nondiffracting properties [1]. These beams were only recently experimentally observed by Siviloglou et al [2]. Airy beams generate a growing interest since they remain focused during the propagation, exhibit properties of self-healing and their trajectory is parabolic along the propagation axis. At present, applications of Airy beams are dedicated to trapping and filamentation. For applications to extreme nonlinear optics and material structuring, nondiffracting beams have key benefits over gaussian beams [3].

In this context, we develop a new experimental setup to generate femtosecond Airy beams with micronic spot-size.

Experimentally, Airy beams can be generated by applying a cubic phase on a Spatial Light Modulator (SLM) to an incident Gaussian beam, and then a lens performs a Fourier Transform of this modulated beam. A Airy beam begins, by this way, to propagate from the focal image plane of the lens.

In this work, we propose a step up (Fig. 1 (a)) that combines a long focal lens L with a microscope objective lens (MO). A phase SLM positioned in the focal plane of the lens is illuminated by the gaussian femtosecond beam. The modulation depth of the SLM introduces a bias that has to be filtered. Thus, we add to the cubic phase term a linear phase modulation directly onto the SLM. A spatial filter (Fig. 1 (a)) positioned in

(a)

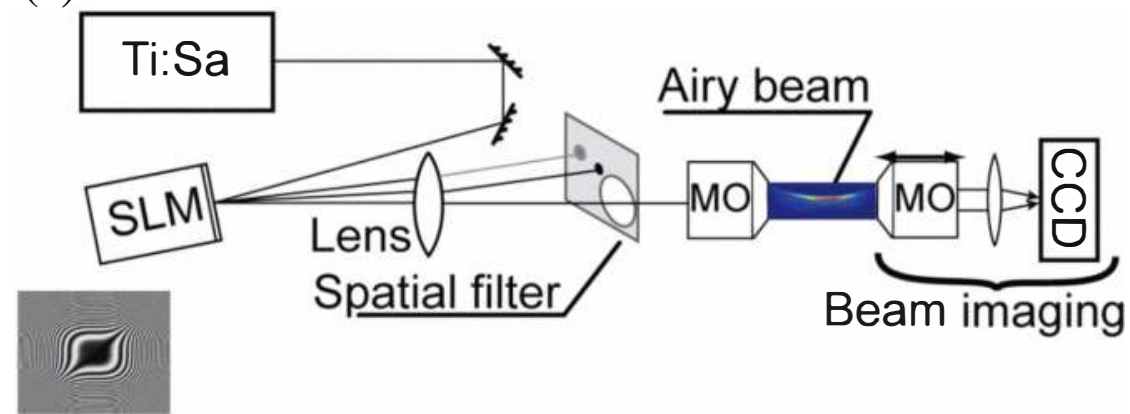

Figure 1 (a) Set-up proposed to realize micro-Airy beam. (b) Micro-Airy beam computed in the transverse xy plane. (c) Micro-Airy beam propagation computed along the $\mathrm{z}$ axis.

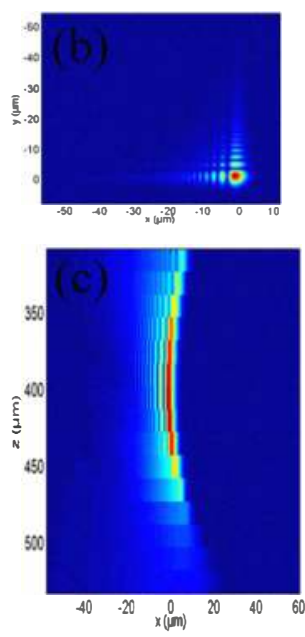

the focal plane of the lens $\mathrm{L}$ is used in order to select the first diffraction order. A micro-Airy beam is then obtained after the microscope objective (MO). First numerical simulations, based on angular spectrum of plane waves, shows (Fig 1 (b). and (c)) that, with our set-up, a micro-Airy beam of $2 \mu \mathrm{m}$ of central lobe diameter propagates along a distance of $120 \mu \mathrm{m}$ with a lateral shift of $7 \mu \mathrm{m}$.

Numerical simulations including the modulation depth of the SLM, spatial filter and femtosecond pulses will be presented, and first experimental results will be also reported. This works opens new perspectives for nonlinear propagation of highly focused Airy beams.

\section{References}

[1] M.V.Berry, N.L.Balazs, 'Nonspreading wave packets," Am. J. Phys. 47, (1979)

[2]G. A. Siviloglou, J. Broky, A.Dogariu and N.D.Christodoulides,'”Observation of Accelerating Airy Beams,' 'Phys. Rev. Lett, 99, 213901, (2007)

[3] M. K. Bhuyan, F. Courvoisier, P.-A. Lacourt, M. Jacquot, R. Salut, L. Furfaro, and J. M. Dudley "High aspect ratio nanochannel machining using single shot femtosecond Bessel beams," Appl. Phys. Lett. 97, 081102 (2010). 